\title{
L'ÉVOLUTION DES TERMES ET EXPRESSIONS DÉSIGNANT LES TESTICULES, DES VALSEUSES DE BERTRAND BLIER (1972-74) À NOS JOURS
}

\author{
Laurent CANAL \\ Université Masaryk, Brno
}

\begin{abstract}
En): This article offers a comparison between the terms designating the testicles in Bertrand Blier's work Les Valseuses (the novel, the film and the film trailer) from the early 1970s, and the last ones listed in various recent dictionaries, paper and online. These synonyms, attested in writing (for an approximate dating of their use), follow variations: diaphasic, diatopic and diachronic; have functions: playful and bypassing taboo; and one, or even several common semantic matrices. Their frequency in writing in a contemporary web corpus, can allow us to distinguish the terms "aged" (or obsolete) from those which are "classic" (common and transgenerational) before comparing them to "contemporaries", especially associated with young people from the cities.
\end{abstract}

Keywords (En): Les Valseuses; argot; testicles; synonyms; variations; functions

Mots-clés (Fr) : Les Valseuses ; argot ; testicules ; synonymes ; variations ; fonctions

DOI : $10.32725 /$ eer.2021.011

\section{Introduction}

En français, aucune partie du corps humain ne semble échapper à une, voire à de nombreuses expressions idiomatiques. Pour ce qui est des parties intimes, notamment en argot, la pléthore des termes est remarquable concernant le sexe «[c]omme dans tous les argots [...], vu l'importance particulière qu'on lui accorde. » (BĂLĂ, 2011: 37). Les fonctions d'un tel lexique ne reflètent pas vraiment celle qui est généralement considérée comme l'alpha de l'argot: la fonction cryptique. En effet, la fonction ludique et celle de contournement de tabou, sont très présentes et semblent même s'agréger dans certains cas. Mais ces tabous relèvent moins d'un interdit imposé par la société que de la gêne ou la honte que la pudeur ou la décence de chacun exigent quand il s'agit d'évoquer des termes relatifs à la sexualité de façon explicite. Les testicules, attributs masculins, sont souvent le pivot d'expressions populaires courantes et objets d'un grand nombre de métaphores, euphémismes, etc. soumis aussi à des variations diastratique, diatopique, diaphasique et diachronique.

Les Valseuses, œuvre littéraire (1972) et cinématographique (1974) de Bertrand Blier (cinéaste incontournable et fils de l'un des comédiens les plus populaires du cinéma français : Bernard Blier, 1916-1989), dont les succès notables témoignent d'une époque et d'un état d'esprit post-soixante-huitard, empreint de contestation, de révolte et de libération, sexuelle notamment, est représentative pour ce présent travail qui consiste à en prélever les termes orchidiens (dans le roman, le film et la bande-annonce particulièrement), à situer leur origine géographique, leur étymologie, la date qui peut leur être attribuée (dans les dictionnaires papier et en 
ligne), et les distinguer selon leur fréquence dans un corpus électronique récent. Ces informations peuvent nous donner une idée de leur longévité, de leur évolution et éventuellement nous permettre de déterminer ceux qui ont perduré (transgénérationnels) et ceux qui sont vieillis (ou obsolètes). Et enfin, nous les confronterons avec ceux qui ont été attestés plus récemment, du verlan et dérivés essentiellement, afin de dégager d'éventuelles contiguïtés et spécificités.

\section{Le corpus du roman Les Valseuses (1972)}

Au tournant de la libération sexuelle post-soixante-huitarde, dans un contexte pompidolien, a priori plus permissif, Bertrand Blier écrit son premier roman : Les Valseuses, qui relate les errances de deux jeunes voyous idiots en rupture avec la société. Le titre très accrocheur et transgressif qui évoque deux «couillons » en mouvement (Jean-Claude et Pierrot) reflète bien l'histoire.

L'argot parisien « classique », décrit par Denise FRANÇOIS-GEIGER (1990: 8), perpétué notamment par les textes de Michel AUDIARD (1920-1985), est aussi celui que Bertrand Blier a souvent articulé dans la communication avec son entourage et à travers les scénarios à partir desquels il donnait la réplique à son père, et qui ne se dissocie pas d'une tonalité et d'une musicalité que l'on retrouve dans son œuvre.

Dans le roman, les synonymes de valseuses (qui, bien que typique de cet argot, n'apparaît qu'en titre) et expressions associées sont nombreux mais celui qui est le plus utilisé est le terme couilles. Les noms, mots composés et expressions qui dérivent de ce terme sont divers et variés : couillons «idiots» (p. 399), couille molle « pleutre» (p. 92), casse-couilles « personne énervante» (p. 203); partir en couille « mal évoluer» (pp. 393, 412), sur une couille de [midi] « vers, à environ » (p. 371). Le verbe couillonner « tromper », est aussi présent (p. 128).

Nous trouvons aussi des expressions implicites où le terme est suggéré : se les geler « avoir froid» (pp. 85, 182), les briser « agacer» (pp. 76, 315), où je pense (p. 174), les parties (pp. 115, 380) ou dans la phrase: «Si jamais on doit me les couper [...] je lui ferai bouffer les siennes » (p. 20), et une expression verlanisée : balle-peau: «Mollo ! [...] Sinon ce soir vous ferez balle-peau !...» (p. 333), de peau de balle «rien", dans la locution verbale faire peau de balle, synonyme de faire tintin, dans le sens de « jeûner, ne rien obtenir » ( $A B C$ de la langue française, en ligne, abr. AB). Une expression ambiguë citée par le personnage féminin, MarieAnge : «Mon paillasson c'est pas pour ses boots!» (p. 300) peut aussi retenir l'attention.

Et enfin, les synonymes de valseuses : testicules (pp. 32, 193), rognons (p. 181), bougnes (p. 264), noix (p. 379), burnes (pp. 180, 407), grelots x2 (pp. 27, 181), bourses (pp. 370, 414), et enfin couilles : 23 occ. (pp. 17, 18 (x2) $20,21,32^{(\mathrm{x} 2)}, 35^{(\mathrm{x} 2)}$, $\left.49,75,76,82,84,92^{(\times 2)}, 93,117,125,139,280,315,429\right)$ exception faite des dérivés supra.

\section{Le corpus du film Les Valseuses (1974)}

Deux ans après son scandale littéraire, Blier réalise le film, un road movie en réaction au cinéma bourgeois, irrévérencieux et drôle, qui fera sa notoriété ainsi que 
celles des acteurs Gérard Depardieu (Jean-Claude) et Patrick Dewaere (Pierrot). Le film est toujours très visionné même par les jeunes générations.

Le terme valseuses qui était déjà vieilli dans les années 1970 a un avenir prometteur grâce au film même si son emploi reste limité ${ }^{1}$. Mais les dialogues du film ne présentent que deux synonymes de valseuses : testicule (dans le diagnostic du docteur) et couilles, majoritairement utilisé dans le roman. Et une expression : tu nous les brises (briser les noix ${ }^{2}$ : ennuyer), utilisée par Marie-Ange (Miou-Miou), le personnage féminin principal, pour rabrouer Carnot, un personnage secondaire, qui se plaint de sa frigidité : "Qu'est-ce que tu nous les brises ? $^{3} t^{\prime} e s$ pas heureux? t'as pas eu ce que tu voulais? ". Cette masculinisation implicite du discours correspond en partie à un comportement linguistique d'homme proche de celui relevé dans le film L'Esquive (KECHICHE, 2004) par Sabine Bastian (2009 : 17-18) qui souligne notamment la difficulté ou l'impossibilité de traduire une expression telle que «T'en bats $[\mathrm{sic}]$ les couilles » dans une autre langue.

Les tentatives de traduction du titre Les Valseuses, qui porte divers sémantismes (testicules, idiots, inséparabilité, mouvement), rencontrent aussi un problème de conception. Des traductions ne prennent en compte qu'un seul de ces sémantismes : Jaja [pol] « les œufs », Riipukset [fin] « les pendentifs » : métaphores de testicules ; ou adaptent uniquement l'idée de mouvement : Вальсирующие [rus], Вальсуючі [ukr], Валсиращите [bul] «valse»; d'autres adaptations sont plus libres et assez insolites : Going Places [eng] «lieux de passage » (qui peut aussi s'interpréter selon le contexte comme : « commencer à avoir du succès, aller loin » ou « en voyage $4^{4}$ ), مكان رفتن [per] « l'endroit où aller », Die Ausgebufften [ger] « les rusés », Los rompepelotas [spa] « les casses-couilles», I santissimi [ita] « le plus saint» et surtout Buzíci [cze] "les pédés»; ou ont choisi une transcription phonétique: バルスーズ [jpn] /ba.ru.see.zu/. Mais aucune ne communique vraiment les sémantismes du titre original. Cette problématique démontre que si les images mentales issues d'expressions métaphoriques ont une connotation qui s'assimile en fonction de la conception du réel que se font les locuteurs d'une même communauté linguistique, une traduction mot-à-mot nécessite une représentation partagée de ces images. Et l'argot, qui utilise aussi des métaphores permettant de communiquer plusieurs idées à partir de quelques mots, voire d'un seul, a un aspect pratique qui favorise et légitime son utilisation.

\footnotetext{
${ }^{1}$ Nous le trouvons ponctuellement dans le rap : Ménélik (2000), Neshga et JeanJass (2015) ou Douze (2016) qui utilisent Les valseuses comme titre ; idem dans le black metal : Grand Guignol Orchestra (2019); etc.

${ }^{2}$ Cette expression est communément admise comme un euphémisme de casser les couilles, toutefois nous rencontrons dans le film une autre utilisation de noix dans l'expression: coller aux noix (au cul: être aux trousses de), il est donc possible qu'il y ait eu un glissement sémantique de noix (fesses), que l'on retrouve encore dans se casser les noix (se casser le cul: se donner du mal), à noix (testicules) dans briser les noix (casser les couilles : importuner, agacer).

${ }^{3}$ Dans le roman, l'expression est plus neutre : «Qu'est-ce que tu nous emmerdes? » (BLIER, 1972 : 37).

${ }^{4}$ L'affiche américaine, qui montre une scène d'autostop, suggère surtout l'aspect road movie du film.
} 


\section{Le corpus de la bande-annonce du film}

La bande-annonce de F.A. Reznikov qui précédait le film, une suite de dessins / collages (un bonhomme schématisé dont les attributs sont cachés par une succession d'images d'objets divers) sur lesquels le narrateur (Claude Piéplu5) cite simultanément dans un monologue toute une liste de mots d'argot et d'expressions (pas toujours en rapport avec les collages) synonymes du terme valseuses, nous donne un exemple de la variété de termes ${ }^{6}$ pouvant coexister dans une époque donnée :

En général, une feuille de vigne, ça cache quelque chose. Certains préfèrent le drapé antique. D'autres n'hésitent pas à bafouer la nature. Et pourtant! Et pourtant ! ça existe. Ça porte un nom. Étant donné que celui qui a des roubignoles ne peut pas ressembler à celui qui a des balloches, imaginons ceux qui ont des roustons, des roupettes, des boules, des bugnes, des boots, des burnes, des bonbons, des burettes, des ronfles, des rouleaux, des précieuses, des joyeuses, des baladeuses, des glorieuses, des paresseuses, des flambeuses, des olivettes, des montgolfières, des castagnettes, des délinquantes, sans oublier... les bijoux de famille, les voisines du dessous, les deux orphelines, et surtout, et surtout! Les valseuses. Qu'il ne faut pas confondre avec le valseur ${ }^{7}$... Nuance... [...] (Transcription de la bande-annonce)

Les collages dont les objets montrés ne sont pas cités évoquent le même sujet : des inséparables (Agapornis ; oiseaux), bélier, rognons, prunes, raisins et autre fruit. De nombreux végétaux : guignes, mirabelles, noisettes, noix, pruneaux (GUIRAUD, 1993 : 59, 61, 75, 80,112, 113), peuvent être utilisés pour désigner les testicules comme ces derniers peuvent l'être pour désigner des végétaux tels que l'orchidée dont les racines grecques permettent de créer un pseudo-terme scientifique: orchidoclaste « casse-couilles » (Ta vamp orchidoclaste, Hubert-Félix THIÉFAINE, 2011). Comme le signale Jean-Louis Calvet, avec l'équation argent = nourriture et la problématique de l'étymologie de pognon (CALVET, 1990 : 48) :

1) le sens de la dérivation n'est pas nécessairement unilatéral

2) cette équation ne se limite pas à un registre particulier.

\section{Discrimination des synonymes}

Afin de ne pas dépasser le cadre de ce présent article, nous avons exclu les termes précieuses (1955, AB ; 1957, Dictionnaire de l'argot de J.-P. Colin, abr. DA), joyeuses (1881, DA ; 1947, AB), rouleaux (1876, DA ; 1890, AB) et burettes $(1889, \mathrm{DA} ; 1899, \mathrm{AB})$ qui ne sont que très peu utilisés aujourd'hui et qui n'appartenaient pas vraiment dans les années 1970 à une jeune génération. De même pour olivettes (1955 olives, DA) et bonbons (1880, DA) qui sont généralement utilisés comme euphémismes à l'instar de nombreuses analogies dans certains contextes qui nécessitent un polissage de la parole (en présence d'enfants par exemple), ce qui révèle aussi une variation diaphasique bien réelle. Aussi, certains synonymes de valseuses ne sont pas recensés comme tels dans les dictionnaires

\footnotetext{
${ }^{5}$ Acteur français et voix célèbre de Les Shadoks, série animée populaire diffusée de 1968 à 1973 sur la première chaîne de l'ORTF : Office de radiodiffusion-télévision française.

${ }^{6}$ En italique dans la transcription.

${ }^{7}$ Valseur désigne « les fesses ».
} 
utilisés : les analogies : boules $^{8}$, bougnes/bugnes : forme provençale de beignet dans le Larousse en ligne (abr. LL), boots ${ }^{9}$, castagnettes; et les noms au féminin pluriel glorieuses, flambeuses, paresseuses, délinquantes, semblent être des tournures « audiardesques» qui sous-entendent une certaine forme de vanité et évoquent l'immoralité (religieuse et civile), esprit dont les dialogues de Michel Audiard témoignent. Nous pouvons également isoler des termes qui correspondent non pas aux testicules mais à la prostitution : baladeuse (fille des rues, 1852, DA), ronfle (prostituée, 1876, AB) et montgolfière (prostituée nymphomane, 1960, DA). Et enfin, pour les mêmes raisons évoquées ci-dessus, nous avons aussi écarté les termes du livre : bougnes, bourses, grelots et noix.

Quant aux expressions : les deux orphelines «peut-être inspirée du roman de d'Ennery 'les Deux Orphelines' (1874), 1977 [Caradec]»(DA); les bijoux de famille « pénis et testicules » (Le Petit Robert, abr. PR); et les voisines du dessous ; d'origines incertaines ou inconnues, elles semblent avoir été choisies pour leur fonction ludique.

Le corpus électronique French Web 2017 (frTenTen17) sur le site sketchengine.eu (abr. CF) qui comprend plus de 5,7 $\mathrm{M}^{\mathrm{ds}} \mathrm{d}$ 'occurrences, nous permet d'avoir un aperçu fréquentiel des termes choisis apparaissant à l'écrit sur Internet.

\begin{tabular}{|l|c|}
\hline \multicolumn{1}{|c|}{ Expressions } & occurrences CF \\
\hline bijoux de famille & 283 \\
\hline deux orphelines & 14 \\
\hline voisines du dessous & 1 \\
\hline
\end{tabular}

Tableau 1 : Les expressions de la bande-annonce

Les deux autres acceptions de bijoux de famille: «qui se transmettent d'une génération à l'autre » (PR), et « biens immobiliers de l'État » (LL), totalisant 602 occ. sur 885 locutions, ont été écartées de notre recherche comme tous les bruits des termes et expressions traités. Mais nous pouvons supposer que la récurrence de l'expression relative aux testicules est probablement entretenue et véhiculée par l'usage des deux autres. Elle associe au moins deux idées sur les génitoires : l'objet précieux et « la transmission » (la procréation), et peut-être aussi l'orgueil.

L'expression les deux orphelines a peut-être pu subsister grâce aux œuvres du même nom en livres et nombreuses adaptations à l'écran (petit et grand). Et enfin l'expression les voisines du dessous, peu usitée, semble aussi être une tournure de dialoguiste. Les trois semblent vieillies mais nous pouvons constater que les bijoux de famille, qui est certainement la plus ancienne et la plus utilisée aujourd'hui, a été transmise de génération en génération au-delà du cadre familial. Les deux autres sont plutôt obsolètes, surtout les voisines $d u$ dessous, certainement la plus récente mais aussi plus proche de l'hapax que de l'expression populaire.

En ce qui concerne le terme couilles, certainement trop vulgaire (dans tous les sens du terme) pour une stratégie promotionnelle efficace, il n'apparaît pas dans la

${ }^{8}$ Le terme boule : «(fondement, postérieur, cul) $\leftarrow$ dialecte kalderash (tsigane) [...] 》 (CALVET, 1993, dans GoudAillier, 2019: 19,75), comme noix, a peut-être subi un glissement sémantique par analogie.

${ }^{9}$ Qui corrobore néanmoins la citation du livre (supra). 
bande-annonce. Toutefois, il est cité comme synonyme de valseuses dans le film et de nombreuses fois dans le livre. Nous analyserons donc les termes balloches, burnes, couilles, roubignoles, roupettes, roustons et valseuses.

\section{Problématiques des étymologies, datations et origines géographiques}

Le PR nous donne l'étymologie de roubignoles : «1862; provenç. roubignoli "testicules", issu de robin, surnom du bélier ${ }^{10} »$. Et pour balloches (ou baloches), qui n'est pas dans le PR, le DA nous donne l'étymologie suivante : « déverbal de ballocher, osciller en pendant, mot picard ». Selon Calvet, «bien des innovations lexicales viennent de la capitale et pénètrent lentement en province, mais l'inverse peut se produire [...]» (CALVET, 1990: 42). Ces deux premiers exemples le confirment et suivent aussi un «parcours diatopique [...] pour accéder à l'argot » (CALVET, 1990 : 44).

Pour ballocher, le Trésor de la Langue Française informatisé (TLFi, abr. TL) nous donne la définition suivante : "Argot: être secoué, balloté », et pour l'étymologie : "flâner, paresser », ce qui nous renvoie à baladeuses et paresseuses. Le terme balloté semble nous proposer un étymon : balle : « [1268]. Gros paquet de marchandise généralement enveloppé dans une toile et lié de cordes »(PR), synonyme de «sac». Ce terme contemporain du verbe de l'ancien français balochier (XIII siècle) : " se balancer, pendre » (TL), a donné emballer, déballer, et notamment ballot, qui signifie aussi «couillon», balourd ou encore baluche (ballot, dans le même sens, + le suffixe -uche) pour «sot» (GUIRAUD, 1963 : 48). Et balloches (ballot + le suffixe -oche) pourrait peut-être se différencier de baloche (bal [dansant] + -oche), à moins que ce dernier fasse référence à la prunelle : «baloches ou blosses [...] (anc. Franç., beloces [...])» (GUIRAUD, $1963: 65$ ), ce qui nous donnerait peut-être d'autres indications: balosses en ChampagneArdenne, bloche en Normandie (HUMBERT, 1852: 44) et bolosses en Bretagne ou bolosen et bolos ${ }^{11}$ (DU RUSQUEC, 1895, en ligne). Quoi qu'il en soit, de ballot (peutêtre même baltringue) à baluchon (baluche + le suffixe diminutif -on) en passant par les termes botaniques régionaux désignant une prune, l'idée d'" osciller en pendant » (supra) tel un sac «trimballé » ou un fruit «ballottant» au gré du vent est évoquée par analogie. Il est donc probable que diverses sources sont à l'origine de balloches. Par ailleurs, il peut s'agir également d'un fruit qui pousse sur un ballochier : sorte d'oranger (RAYMOND, $1835: 75$ ). Et balonche, «balance» en picard, qui évoquerait l'équilibre entre deux éléments identiques en suspension est une métaphore plausible.

Certaines étymologies, même en excluant les plus fantaisistes, peuvent donc se reposer sur plusieurs sémantismes et paraître a priori assez "bringuebalantes », d'où l'intérêt d'établir « le protosémantisme à partir de quoi on cherche quelque mot dans lequel il a pu s'actualiser » (CALVET, 1990: 46). Toutefois, les équations testicules $=$ mépris (ballot, couillon, etc.), testicules $=$ mouvement, et testicules $=$ fruit, semblent se renvoyer l'une à l'autre.

\footnotetext{
${ }^{10} \mathrm{D}$ 'où, certainement, l'idée du bélier dans la bande-annonce.

${ }^{11}$ Terme breton que l'on pourrait ajouter aux étymologies possibles de l'actuel bolos.
} 
Roubignoles (supra), roupettes, probablement de roupille, «dialectal pour "guenille", [...]» (TL), roustons « du languedocien roustoun » dans le LL, «peutêtre issu de roustio qui, dans le Dauphinois, désigne une châtaigne rôtie » (CALVET, 1993 :111), ainsi que rouleaux, semblent aussi avoir le même étymon: roue : « organe de forme circulaire [...]» (LL). Et burnes, du rouchi, patois picard, qui « désigne le nœud d'un arbre »(CALVET, 1993 : 111), comme peut-être rouston, déploie un peu plus le lexique lié à la flore. Mais nous pouvons remarquer que roubignole, roupette, rouston et rouleau ne s'emploient pas pour désigner un imbécile alors que burne, voire grosse burne est souvent utilisé dans ce sens. Ce qui signifie que l'interpénétration sémantiques des équations supra a des limites intéressantes à définir pour affiner les sens et les emplois de ces prétendus synonymes.

\section{Occurrences des termes choisis}

L'ensemble de ces termes plus ou moins anciens retenu de Les Valseuses, essentiellement de la bande-annonce, peut être classé selon un ordre chronologique en fonction des datations dans l'AB et dans le DA. Une comparaison des fréquences entre tous ces synonymes à partir du $\mathrm{CF}$, peut nous permettre de distinguer les plus utilisés dans la communication écrite.

\begin{tabular}{|l|l|r|}
\hline \multicolumn{1}{|c|}{ Lemmes } & \multicolumn{1}{|c|}{ dates } & occurrences CF \\
\hline couilles & $1178(\mathrm{DA})$ & 28071 \\
\hline roupettes & $1779(\mathrm{DA}) ; 1790(\mathrm{AB})$ & 117 \\
\hline balloches & $1836(\mathrm{AB}) ;(\mathrm{DA})$ & 137 \\
\hline roustons & $1836(\mathrm{AB}) ;(\mathrm{DA})$ & 147 \\
\hline roubignoles & $1836(\mathrm{DA}) ; 1862(\mathrm{AB})$ & 200 \\
\hline burnes & $1888(\mathrm{AB}) ; 1888(\mathrm{DA})$ & 160 \\
\hline valseuses (titre du film exclu) & $1952(\mathrm{AB})$ & 160 \\
\hline
\end{tabular}

Tableau 2 : Les termes choisis du livre, du film et de la bande-annonce

Le terme le plus utilisé, deux fois plus que testicules (14 077 occ.), est le plus ancien. Les expressions désignant cette partie du corps n'emploient généralement pas le terme testicules. Le nombre d'expressions relatives au terme couille fait la différence. Au pluriel, elles se cassent, se brisent, se pètent, etc. pour ennuyer, importuner, agacer, déranger ; mais on peut s'en battre : s'en foutre, pour mépriser ; on peut même s'en faire en or: gagner beaucoup d'argent; etc. Au singulier, elle peut être synonyme de problème, surtout quand elle est dans le pâté ou dans le potage; mais on peut tout de même l'utiliser comme terme amical avec le pronom $m a$. Depuis le XII ${ }^{\mathrm{e}}$ siècle, couilles s'est développé sous diverses formes, notamment en partant d'une expression pour créer un nom : couille molle ou casse-couilles. Aucun autre terme ne l'a destitué. Évidemment, les autres synonymes peuvent remplacer couille dans bon nombre d'expressions et c'est sans doute ce qui leur permet de subsister en partie, avec la règle de l'euphonie, l'aspect ludique, mais aussi le côté pratique d'une multitude de significations et de registres. Roupettes, balloches, roustons, roubignoles, valseuses sont vieillis mais toujours pas obsolètes 
pour ces mêmes raisons. Burnes et surtout couilles, qui sont de loin les plus utilisés, sont quant à eux assurément transgénérationnels.

L'étymologie de couilles donnée par le DA : «du lat. vulg. Colea, même sens [...] ; symbole de virilité », cité dans le Roman de Renart ( " couille à Joffre »), nous renvoie au Moyen Âge (1178), ce qui fait de ce terme le plus ancien (CALVET, 1993 : 112) et donc le premier en français. Même testicules (qui paraît de ce fait être l'euphémisme de couilles), selon l'étymologie donnée par le TL, est plus récent (1304). Il est emprunté au latin testiculus, dérivé de testis : témoins (de la virilité). Pas étonnant donc que les expressions associées à ces parties du corps soient souvent utilisées pour évoquer le courage longtemps attribué à un signe de virilité : avoir des couilles : être courageux; en avoir au cul, ou comme des ananas (AB), la grosseur du fruit évoquant la grandeur du courage. Pour comparer ces termes, nous pouvons rajouter les "néologismes ${ }^{12}$ » qui apparaissent dans la dernière édition (2019) de Comment tu tchatches! Dictionnaire du français contemporain des cités de Jean-Pierre Goudaillier (abr. FC). Ces termes (yeucous, yeucs, yeuk et balek) sont tous issus de couilles.

\begin{tabular}{|c|c|c|}
\hline Lemmes & dates & occurrences CF \\
\hline \multicolumn{3}{|c|}{ verlan de couilles } \\
\hline yeucous & $2001,2019(\mathrm{FC})$ & 2 \\
\hline yecou & - & 5 \\
\hline yeukous & - & 1 \\
\hline \multicolumn{3}{|c|}{ apocope du verlan } \\
\hline yeucs & 2001,2019 (FC) & 0 \\
\hline yeuc & - & 3 \\
\hline yeuk & 2019 (FC) & 3 \\
\hline yeuks & - & 13 \\
\hline \multicolumn{3}{|c|}{ (expression) aphérèse + apocope } \\
\hline balek & $2019(\mathrm{FC})$ & 121 \\
\hline balec (52), ballec (21), ballek (4) & - & $(77)$ \\
\hline
\end{tabular}

En ce qui concerne ces néologismes, seul balek, issu de l'expression s'en battre les couilles, semble avoir un certain succès, mais les verlans de couilles (toutes graphies confondues) sont très peu utilisés à l'écrit. L'expressivité et la compréhension d'un terme (ou locution) relatif aux testicules, souvent essentielles et indissociables, qui excluent généralement le cryptage, et l'usage fondamentalement oral du verlan peuvent expliquer en partie cette carence à l'écrit.

\section{Conclusion}

L'ancienneté d'un terme ne signifie donc pas un vieillissement ni une diminution de son utilisation, et inversement, sa nouveauté relative ne traduit pas une utilisation importante. Le plus vieux, le plus utilisé et a priori le plus vulgaire,

\footnotetext{
${ }^{12}$ Le verlan étant essentiellement oral, il est fort probable que ces termes ont été créés de manière
} spontanée dès l'invention du verlan, car couille était populaire et les expressions associées aussi. 
s'est développé et s'est actualisé dans de nombreuses formes et expressions, ce qui a certainement contribué à sa pérennisation.

Dans une formule implicite, où le terme n'est que suggéré, l'utilisation du féminin n'évoque sûrement pas celui de «testicules ». "Ça m'en touche une sans faire bouger l'autre », formule chiraquienne reprise en 2018 par Macron ${ }^{13}$ en est un exemple qui peut résumer la complexité de ce lexique : il révèle une variation diastratique (et aussi diaphasique) et deux fonctions : ludique et de contournement de tabou, qui s'interpénètrent, l'humour permettant de palier légèrement la gêne que la pudeur implique. Cette expression «présidentielle», comme l'expression contractée balek, dépend d'une double matrice sémantique (le mépris et le mouvement) qui peut altérer son universalité, à l'instar de valseuses. Certes des matrices universelles relatives à la forme ou la sustentation existent, comme jaja en polonais ou son pendant finnois Riipukset, mais les images partagées liées aux testicules, surtout lorsqu'elles se cumulent, concrètes et multiples, semblent complexifier considérablement les comparaisons avec les autres langues. Peut-être faudrait-il les distinguer des matrices abstraites comme celle de «l'argent» dont l'utilisation universelle primaire, se nourrir, permet un rapprochement plus évident avec d'autres langues ${ }^{14}$.

Les synonymes choisis pour ce travail, qui découlent aussi de ces facteurs, entre autres, ont souvent une étymologie incertaine (voire multiple) et une datation approximative de leur utilisation qui dépendent d'attestations écrites essentiellement, ce qui ne facilite pas le traçage de leur parcours diatopique et diachronique essentiellement oral.

Les Valseuses, œuvre irrévérencieuse qui a marqué à jamais son époque, la révolution sexuelle des années 1960-1970 et les cinquante ans qui ont suivi n'ont pas effacé des siècles de pudicité, voire de pudibonderie. Désigner les parties intimes du corps à l'aide d'un langage détourné, dépourvu de connotations associées aux mots du registre concret, est une pratique séculaire qui demeure, encore aujourd'hui et malgré une libération de la parole avérée, bien ancrée.

\section{CORPUS UTILISÉS}

Roman : BLIER Bertrand (1972), Les Valseuses, Paris, Robert Laffont.

Film : BLIER Bertrand (1974), Les Valseuses, France, CAPAC, UPF, SN Prodis.

Bande-annonce du film : REZNIKOV F. A. (1974), B. A. Les Valseuses, France.

\section{RÉFÉRENCES BIBLIOGRAPHIQUES}

BĂLĂ Laurenţiu (2011), Le corps humain en argot: le sexe, in: Analele Universităţii din Craiova, Ştiinţe Filologice, Limbi Străine Aplicate 1.

BASTIAN Sabine (2019), La traduction des argots 'jeunes' - l'exemple du film L'Esquive et ses traductions allemandes, in: Standard et périphéries de la langue, Oficyna Wydawnicza LEKSEM.

CALVET Jean-Louis (1994), L'argot, éd. 2007, Paris, PUF.

${ }^{13}$ Theveniaud, Le Parisien, 2018, en ligne.

${ }^{14}$ Cf. Calvet, L'argot, (1994 : 62). 
CALvet Jean-Louis (1993), L'argot en 20 leçons, ou, Comment ne pas en perdre son français : suivi d'un appendice grammatical, Paris, Payot \& Rivages.

CALVET Jean-Louis (1990), L'argot comme variation diastratique, diatopique et diachronique, in: Parlures argotiques, FranÇOIS-GEIGER Denise, GOUDAILLIER Jean-Pierre (éd.), Langue française 90, Larousse, Paris.

Colin Jean-Paul, MÉvel Jean-Pierre (1990), Dictionnaire de l'argot, éd. 1993, Paris, Larousse.

FranÇOIS-GEIGER Denise (1990), Panorama des argots contemporains, in: Parlures argotiques FRANÇOIS-GEIGER Denise, GOUDAILLIER Jean-Pierre (éd.), Langue française 90, Paris, Larousse.

GUIRAUD Robert (1993), Faune et Flore argotiques II. Flore, Paris, Le Dilettante.

GUIRAUD Robert (1981), L'argot tel qu'on le parle, dictionnaire illustré d'argot moderne, Paris, Jacques Grancher éditeur.

GUIRAUD Pierre (1963), L'argot, Paris, PUF.

GoudAILliER Jean-Pierre (2001), Comment tu tchatches! Dictionnaire du français contemporain des cités, éd. 2019, Paris, Maisonneuve \& Larose.

HUMBERT Jean (1852), Nouveau glossaire genevois, tome1, Genève, Julien frères, en ligne :

https://gallica.bnf.fr/ark:/12148/bpt6k50673v/f75.item.texteImage, consulté le 20.12.2020.

RAYMOND François (1835), Supplément au dictionnaire de l'Académie française, Sixième édition, Paris, Gustave Barba, en ligne :

https://books.google.cz/books/about/Suppl\%C3\%A9ment_au_Dictionnaire_de_1_ Acad\%C3\%A9m.html?id=kkgT1bv6fx0C\&redir_esc=y, consulté le 10.1.20 $\overline{2} 1$. ROBERT Paul (2020), Le nouveau Petit Robert, Paris, Édition du Petit Robert.

DU RUSQUEC Henri (1895), Nouveau Dictionnaire pratique et étymologique du dialecte de Léon, avec les variantes diverses, dans les dialectes de Vannes. Tréguier et Cornouailles, Paris, E. Leroux. En ligne: https://gallica.bnf.fr/ark:/12148/bpt6k58316694/texteBrut, consulté le 20.12.2020.

THEVENIAUD Pauline (2018), Au Salon, Macron la joue cash avec les agriculteurs, LeParisien.fr. Mis en ligne le 25 février 2018: https://www.leparisien.fr/politique/au-salon-macron-la-joue-cash-avec-lesagriculteurs-25-02-2018-7577868.php, consulté le 20.1.2021.

\section{Sites Internet}

$\mathrm{ABC}$ de la langue française: http://www.languefrancaise.net/Bob/Introduction, consulté le 2.1.2021.

Trésor de la Langue Française informatisé (TLFi) : http://atilf.atilf.fr/, consulté le 2.1.2021.

Le Dictionnaire de la Zone: https://www.dictionnairedelazone.fr/, consulté le 2.1.2021.

Le Larousse en ligne : https://www.larousse.fr/dictionnaires/francais, consulté le 2.1.2021.

Sketch Engine : https://www.sketchengine.eu/, consulté le 2.1.2021.

$$
-138-
$$


YouTube : Bande-annonce du film Les Valseuses montée par F.A. Reznikov :

https://www.youtube.com/watch? $v=$ pUnMWTN5R8g\&feature=emb, consulté le 2.1.2021. 